\title{
Physical Activity After Surgery for Severe Obesity: The Role of Exercise Cognitions
}

\author{
Eveline J. Wouters • Junilla K. Larsen • \\ Hanna Zijlstra $\cdot$ Bert van Ramshorst $\cdot$ Rinie Geenen
}

Published online: 14 September 2010

(C) The Author(s) 2010. This article is published with open access at Springerlink.com

\begin{abstract}
Background Physical activity after bariatric surgery is associated with sustained weight loss and improved quality of life. Some bariatric patients engage insufficiently in physical activity. This may be due to exercise cognitions, i.e., specific beliefs about benefits of and barriers to physical exercise. The aim of this study was to examine whether and to what extent both physical activity and exercise cognitions changed at 1 and 2 years post-surgery and whether exercise cognitions predict physical activity. Methods Forty-two bariatric patients (38 women, 4 men; mean age $38 \pm 8$ years, mean body mass index prior to surgery $47 \pm 6 \mathrm{~kg} / \mathrm{m}^{2}$ ) filled out self-report instruments to examine physical activity and exercise cognitions pre- and post-surgery.
\end{abstract}

\section{E. J. Wouters $(\bowtie)$}

Department of Allied Health Professions,

Fontys University of Applied Sciences,

P.O. Box 347, 5600 AH Eindhoven, The Netherlands

e-mail: e.wouters@fontys.nl

J. K. Larsen

Behavioural Science Institute, Radboud University Nijmegen,

Nijmegen, The Netherlands

H. Zijlstra

Department of Nutrition and Dietetics,

School of Sports and Nutrition, University of Applied Sciences,

Amsterdam, The Netherlands

B. van Ramshorst

Department of Surgery, St. Antonius Hospital,

Nieuwegein, The Netherlands

E. J. Wouters $\cdot$ R. Geenen

Department of Clinical and Health Psychology,

Utrecht University,

Utrecht, The Netherlands
Results A large increase in physical activity and favorable changes in exercise cognitions were observed after surgery, viz. a decrease of fear of injury and embarrassment and an increase of the perception of exercise benefits and confidence in exercising. Perceiving less exercise benefits and having less confidence in exercising before surgery predicted less physical activity 2 years after surgery. High fear of injury 1 year after surgery predicted less physical activity 2 years after surgery.

Conclusions After bariatric surgery, favorable changes in physical activity and beliefs about the benefits and barriers of exercising are observed. Our results suggest that targeting exercise cognitions before and after surgery might be relevant to improve physical activity.

Keywords Exercise · Morbid obesity · Bariatric surgery Health behavior

\section{Introduction}

People who are physically active have a better physical and mental health than sedentary people [1-6]. Various ways to promote physical activity have been examined [7-9], in particular among overweight individuals considering the weight benefits of increased activity [10, 11]. However, compliance with exercise interventions is a main problem [12]. After the intervention, exercise levels decline and individuals regain weight [13].

Improvement in physical activity has been reported after bariatric surgery for severe obesity, and individuals who are physically active after surgery lose more weight and experience better quality of life compared to those who stay inactive [14]. It is, however, insufficiently clear why some patients engage in sportive activities, while others 
stay sedentary [15]. Theories, such as the self-regulatory model of illness, stress the importance of cognitions as determinants of health behavior [16, 17]. Exercise cognitions, i.e., specific beliefs about benefits of and barriers to physical exercise, correlate to actual exercise behavior, both in general and in overweight populations [18-21]. A prospective study in adolescents revealed favorable changes in cognitions after a weight loss camp [22]. Cognitions about exercise can obstruct or promote physical activity. Physical activity may be hampered by the belief that one might get injured during exercise and by feelings of shame about being observed while doing sports. Physical activity may be promoted by the belief that exercising is beneficial for health or by being confident in sports participation.

Although the importance of physical exercise cognitions and the possible changeability of these cognitions as a result of interventions have been underscored, neither the impact of gastric banding on exercise cognitions nor exercise cognitions as possible determinants of physical activity after surgery have been subject of study. More insight into underlying motivational mechanisms of patients to engage in sportive activities may contribute to tailoring physical exercise programs. The aim of the present study was to examine whether and to what extent exercise cognitions and physical activity change after bariatric surgery and whether exercise cognitions before surgery and 1 year after surgery predict physical activity 2 years after surgery. We hypothesized that exercise cognitions and physical activity change favorably after surgery and-for the exercise cognitions - that perceived benefits of exercise predict more physical activity and that perceived barriers of physical exercise predict less physical activity.

\section{Materials and Methods}

\section{Participants and Procedures}

From November 2000 until April 2004, 156 patients were subjected to laparoscopic adjustable gastric banding at the St. Antonius Hospital Nieuwegein, The Netherlands, using the Lap-Band ${ }^{\circledR}$ system (INAMED Health, Santa Barbara, CA, USA), after screening by a team consisting of a bariatric surgeon, an endocrinologist, a psychologist, and a dietician. Indications for surgery were a body mass index (BMI) $>40 \mathrm{~kg} / \mathrm{m}^{2}$ or a BMI between 35 and $40 \mathrm{~kg} / \mathrm{m}^{2}$ and serious comorbidities. Surgery was performed according to the techniques described by Belachew et al. [23]. Weight and height were measured, and questionnaires were filled out 6 months before and 1 and 2 years after surgery. Of the 156 patients who underwent surgery, 141 attended a preoperative meeting during which they were asked to participate in this prospective questionnaire study. After surgery, two patients underwent a gastric bypass, three patients underwent surgery at a different hospital, 17 withdrew from surgery, three patients withdrew participation due to severe psychopathology, 33 patients did not return the questionnaire at one of the three occasions (before or 1 or 2 years after the operation), and one patient had severe complications and re-surgery, leaving 82 patients. To be able to compare the models examining the prediction from pre-surgery to 2 years after surgery and the prediction from 1 year after surgery to 2 years after surgery, patients with missing values on any of the variables at one of the occasions ( 6 months before and 1 and 2 years after surgery) were excluded. Forty-two patients filled out the questions on physical activity and exercise cognitions completely at all time points. The study protocol received the approval of the hospital Research and Ethics Committee.

\section{Measures}

Weight and height were measured at the hospital with participants wearing light clothes and without shoes and socks. BMI was calculated as weight (kilograms) divided by the squared length (meters).

Physical exercise was assessed with the Baecke questionnaire. This questionnaire yields a validated and reliable sports index [24-26], which is a composite score taking into account the expected energy expenditure for a given sport, the number of hours per week and the number of months per year that one exercises, an estimation of the level of physical activity compared to adults of the same age category as the participant, and the frequency of sweating during leisure time. All kinds of sportive leisure time activity, e.g., walking, swimming, cycling, and dancing, are included.

Perceived benefits of and barriers to physical exercise (exercise cognitions) were measured with the Physical Exercise Belief Questionnaire (PEBQ). This 16-item questionnaire, which is partly based on the Dutch version of the Tampa Scale for Kinesiophobia (TSK) consists of four scales [21]. Two scales assess barriers to physical exercise: "fear of injury" (e.g., "sports are dangerous for me because I easily get injured") and "embarrassment" (e.g., "I feel ashamed of my body when doing sports"). Two other scales measure perceived "exercise benefits" (e.g., "sports are healthy for me") and "confidence" (e.g., "I am a sporty type of person"). The 5-point Likert-rating format ranges from 1 (strongly disagree) to 5 (strongly agree). The psychometric characteristics of the PEBQ have been found to be satisfactory [21]. Cronbach's $\alpha$ values indicate the reliability or internal consistency of an instrument. In the current study, Cronbach's $\alpha$ values 6 months before and 1 and 2 years after surgery were $0.90,0.84$, and 0.90 for fear of injury, 0.90, 0.84, and 0.91 for embarrassment, 0.67, 0.92, 
and 0.70 for exercise benefits, and $0.67,0.81$, and 0.85 for confidence. This suggests a moderate to very good reliability.

\section{Statistical Analyses}

The score distribution of all variables was sufficiently normal to allow parametric statistics. To examine differences in physical activity and exercise cognitions before surgery and 1 and 2 years after surgery, respectively, paired samples $t$ tests were used. Cohen's $d$ effect sizes were computed by dividing the difference between mean scores at two time points by the pooled standard deviations; $d$ values of $0.2,0.5$, and 0.8 are considered small, medium, and large effect sizes, respectively [27]. To examine the stability of cognitions before and after surgery, Pearson correlations were calculated.

Physical activity change scores were calculated by adjusting the physical activity scores 2 years after the operation for baseline physical activity (physical activity 6 months before and 1 year after surgery, respectively) using linear regression analysis. It was examined whether the person's characteristics, BMI, age, and education level were correlated with the outcome variable by correlating these variables 6 months before surgery and 1 year after surgery with physical activity 2 years after the operation and the postoperative change in physical activity. To examine whether exercise cognitions before surgery and 1 year after surgery predicted physical activity 2 years after surgery, we correlated exercise cognitions 6 months before surgery and 1 year after surgery with physical activity 2 years after the operation and the postoperative change in physical activity. Pearson correlations were computed. The alpha level for statistical significance was set at 0.05 . For the paired samples $t$ test, the Bonferroni criterion of 0.02 was used to compensate for multiple testing ( 0.05 divided by the number of three repeated measurements). All analyses were performed using SPSS 17.0 (SPSS Inc, Chicago, IL).

\section{Results}

Thirty-eight (90\%) women and four men participated. They had a mean age before surgery of $38 \pm 8$ years. The highest education level attained by patients was in one case, primary education; in 36 cases, secondary education; and in five cases, tertiary education. The mean BMI before surgery was $47 \pm 6 \mathrm{~kg} / \mathrm{m}^{2}$; the mean BMI values 1 and 2 years after surgery were $37 \pm 7$ and $36 \pm 7 \mathrm{~kg} / \mathrm{m}^{2}$, respectively; and excess BMI loss [28] 1 and 2 years after surgery were $46 \pm 21 \%$ and $53 \pm 25 \%$, respectively. There were no significant differences between the group without missing values and the remainder of the 141 patients with respect to age, gender distribution, baseline BMI, excess BMI loss, exercise cognitions, and physical activity. The mean education level in the group with all measurements was higher than in the remainder of the group $(p=0.01)$.

\section{The Postoperative Change in Exercise Cognitions} and Physical Activity

Six months prior to surgery, ten out of the 42 participants (24\%) engaged in sports activities. One and 2 years after surgery, 29 (69\%) and 28 (67\%), respectively, of the 42 participants participated in sports. Table 1 shows the means and standard deviations of the physical activity scores 6 months before and 1 and 2 years after surgery. One year after surgery, the scores on the Baecke sport index showed increased self-reports of physical activity (the magnitude of

Table 1 Physical activity and exercise cognition scores of 42 patients before and 1 and 2 years after surgery

\begin{tabular}{|c|c|c|c|c|c|c|c|}
\hline Variables & $\begin{array}{l}6 \text { months pre- surgery } \\
\text { Mean (SD) }\end{array}$ & $\begin{array}{l}1 \text { year post-surgery } \\
\text { Mean (SD) }\end{array}$ & $p^{\mathrm{b}}$ & $d^{\mathrm{d}}$ & $\begin{array}{l}2 \text { years post-surgery } \\
\text { Mean (SD) }\end{array}$ & $p^{\mathrm{c}}$ & $d^{\mathrm{d}}$ \\
\hline \multicolumn{8}{|l|}{ Physical activity ${ }^{\mathrm{a}}$} \\
\hline Sport index & $2.0(0.6)$ & $2.5(0.7)$ & $<0.001$ & 0.8 & $2.5(0.7)$ & 0.89 & -0.1 \\
\hline \multicolumn{8}{|c|}{ Exercise cognitions (PEBQ) } \\
\hline Fear of injury & $26.5(8.4)$ & $20.9(8.5)$ & $<0.001$ & -0.7 & $20.0(8.7)$ & 0.70 & -0.1 \\
\hline Embarrassment & $31.9(8.9)$ & $24.1(10.6)$ & $<0.001$ & -0.8 & $24.1(9.7)$ & 0.29 & 0.0 \\
\hline Exercise benefits & $30.8(6.5)$ & $33.6(5.0)$ & $<0.001$ & 0.5 & $33.1(6.1)$ & 0.61 & -0.1 \\
\hline Confidence & $15.0(6.1)$ & $16.8(6.0)$ & $<0.001$ & 0.3 & $16.2(6.4)$ & 0.76 & -0.1 \\
\hline
\end{tabular}

\footnotetext{
${ }^{\mathrm{a}}$ Baecke physical activity questionnaire

${ }^{\mathrm{b}}$ Significance of difference between values 6 months before and 1 year after surgery

${ }^{\mathrm{c}}$ Significance of difference between values 1 and 2 years after surgery

${ }^{\mathrm{d}}$ Effect size
} 
this change was large, $d=0.80$ ). All exercise cognitions were also changed in a favorable direction 1 year after surgery compared to 6 months before surgery. This difference was small for "confidence," medium for "exercise benefits" and "fear of injury," and large for "embarrassment." Compared to the scores after the first year, physical activity and exercise cognitions did not change in the second year after surgery.

The correlation between presurgical "fear of injury" and "fear of injury" 1 year after surgery was $r=0.31(p<0.05)$, and the correlation between "fear of injury" 1 and 2 years after surgery was $r=0.75(p<0.001)$. For "embarrassment," these correlations were $r=0.63(p<0.001)$ and $r=0.68$ $(p<0.001)$, for "exercise benefits," $r=0.56(p<0.001)$ and $r=0.58(p<0.001)$, and for "confidence," $r=0.78(p<0.001)$ and $r=0.76(p<0.001)$. The correlations show that the individual differences were rather consistent with one exception; the prediction of "fear of injury" scores 1 year after surgery from "fear of injury" scores before surgery was modest.

The Predictive Value of Exercise Cognitions Before Surgery

The left part of Table 2 shows the correlations of predictor variables 6 months before surgery with the physical activity level and change 2 years after surgery. The physical activity level before surgery was not significantly correlated with physical activity 2 years after surgery $(p=0.14)$. Obviously, the correlation of physical activity levels before surgery with change in physical activity levels was zero because this change score was statistically adjusted for differences in physical activity levels before surgery.

BMI $(p=0.17)$, age $(p=0.16)$, and education level $(p=0.90)$ were neither significantly correlated with physical activity levels 2 years after surgery nor with change of physical activity during the pre-to-postsurgical time interval ( $p=0.18, p=0.36, p=0.97$ ).

The negative exercise cognitions "fear of injury" $(p=0.14)$ and "embarrassment" $(p=0.72)$ did not correlate with the physical activity sport index 2 years after surgery, but the positive exercise cognitions "exercise benefits" $(p=0.02)$ and "confidence" $(p=0.02)$ were correlated with higher physical activity levels 2 years after surgery. The presurgical score of "exercise benefits" was also correlated with the increase in physical activity from before the operation to 2 years after surgery $(p=0.04)$. Presurgical "confidence" just failed to be significantly correlated to the increase in physical activity $(p=0.05)$.

The Predictive Value of Exercise Cognitions 1 Year after Surgery

The right part of Table 2 shows the correlations of predictor variables 1 year after surgery with the physical activity level and change 2 years after surgery. Physical activity 1 year after surgery was strongly correlated with physical activity 2 years after surgery $(p<0.001)$.

A higher BMI at 1 year after surgery was significantly correlated with reduced physical activity levels $(p=0.02)$ 2 years after surgery, but it was not predictive of the baseline (i.e., at 1 year) adjusted change in physical activity during the second year after surgery $(p=0.16)$.

Age $(p=0.42)$ and education level $(p=0.61)$ were not correlated with physical activity 2 years after surgery or with the change in physical activity during the second year after surgery.

The negative exercise cognition "fear of injury" at 1 year after surgery was correlated with reduced physical activity 2 years after surgery $(p=0.01)$, and this cognition was also a significant predictor of less increase in physical activity during the second year after surgery $(p=0.02)$. The positive
Table 2 Correlations of predictor variables 6 months before surgery and 1 year after surgery with physical activity level and the baseline-adjusted change in physical activity 2 years after surgery

\begin{tabular}{|c|c|c|c|c|}
\hline \multirow[t]{3}{*}{ Predictor variables } & \multicolumn{2}{|c|}{6 months before surgery } & \multicolumn{2}{|c|}{1 year after surgery } \\
\hline & \multicolumn{4}{|c|}{ Physical activity 2 years after surgery } \\
\hline & Level & Change & Level & Change \\
\hline Physical activity & 0.23 & 0.00 & $0.68 * *$ & 0.00 \\
\hline \multicolumn{5}{|l|}{ Person characteristics } \\
\hline Body mass index & -0.23 & -0.22 & $-0.35^{*}$ & -0.23 \\
\hline Age & 0.22 & 0.15 & 0.22 & 0.15 \\
\hline Education level & 0.02 & -0.01 & 0.02 & -0.01 \\
\hline \multicolumn{5}{|l|}{ Exercise cognitions } \\
\hline Fear of injury & -0.23 & -0.15 & $-0.42 * *$ & $-0.37^{*}$ \\
\hline Embarrassment & -0.06 & 0.06 & -0.21 & -0.19 \\
\hline Exercise benefits & $0.36^{*}$ & $0.32 *$ & $0.33^{*}$ & 0.06 \\
\hline Confidence & $0.35^{*}$ & 0.30 & 0.29 & 0.15 \\
\hline
\end{tabular}


exercise cognition "exercise benefits" was correlated to physical activity 2 years after surgery $(p=0.03)$, but it was not predictive for the baseline-adjusted change in physical activity $(p=0.71)$.

\section{Discussion}

After bariatric surgery, physical activity increased and favorable changes in exercise cognitions were observed. Exercise cognitions before surgery and 1 year after surgery predicted physical activity 2 years after surgery.

After surgery, the number of patients who actively engaged in sportive activities almost tripled. Moreover, a decrease in fear of injury and embarrassment and an increase in the exercise benefit belief and confidence were observed, with the largest changes in fear of injury and embarrassment. Because lack of physical activity is a metabolic risk factor independent of body weight, the postsurgical increase in physical activity is a favorable health change, regardless of the weight lost. The observation that also exercise cognitions favorably changed implies that people really change their attitudes towards physical activity.

After bariatric surgery, psychological variables commonly change in a favorable direction [29-31]. It has been found that postsurgical weight loss is not predicted by presurgical psychological variables, but rather, that the operation is necessary to discover who will need extra psychological support [21, 32-35]. Our results do not support these findings for the prediction of physical exercise from preoperative exercise cognitions. Exercise promoting cognitions before surgery turned out to be predictors of physical activity after surgery. Preoperatively, not regarding oneself a sportive type of person and not perceiving physical exercise as something beneficial for health were predictive for a low amount of exercise behavior about 2 years later. Many obese people once experienced bullying when performing physical exercise at school [36], and most obese people do not experience much pleasure from physical exercise [37]. This may have settled low confidence in sportive capabilities and dislike of sports as a rather stable characteristic. Indeed, "exercise benefits" and "confidence" showed less change in the period from pre- to post-surgery than the other cognitions and these presurgical cognitions turned out to be predictive for physical activity several years later. A possible clinical implication of our finding is that postoperative physical activity could be improved by presurgical interventions targeting the belief that exercising is not beneficial for health and the low confidence in sports participation.

The negative cognitions "fear of injury" and "embarrassment" showed larger changes after surgery compared to the positive cognitions. Fear to get injured as a result of physical exercise 1 year after the operation predicted reduced physical activity in the second year after surgery. The role of fear in restraining from physical activity has been extensively examined in patients with chronic pain and is explained by the fear-avoidance model [38]. This model explicates that if pain is (mis)interpreted as being threatening, avoidance of physical activity results and this subsequently has a negative impact on musculoskeletal function, physical performance, and fitness [39]. The present study confirms the suggestion that comparable to chronic pain patients and the findings in sedentary but otherwise healthy overweight adults [40], fear of injury can be a barrier to engage in physical activity and a contributor to the sedentary state of patients after bariatric surgery. When someone avoids physical activity due to fear, the fear cannot extinguish. To deal with fear of injury, it is recommended to carefully choose and gradually buildup exercise programs [37, 41] with little risk for injuries and much pleasure for patients. Programs in water such as aquajogging can be suitable for this purpose [42], but also lifestyle activities that are not directly related to regular sports can be applied such as walking, biking, or dancing.

There are some strengths and limitations to our study. A strength is the longitudinal design, which allowed the prediction of physical activity from exercise cognitions. A limitation is the high proportion of incomplete questionnaires leaving a small number of participants with a relatively high education level, which hampers generalizability of the findings. Although on a group level no changes in the second year after surgery were observed, and the second year is considered an important year in which weight maintenance rather than weight loss is the aim, another limitation might be the relatively short follow-up time of 2 years after surgery. Finally, as an adjunct to the self-report measures of physical activity including all activities of daily life, objective monitoring of physical activity with actometers, or accelerometers could be used in replication research of the current research questions.

In conclusion, physical activity and exercise cognitions substantially improve after surgery. Not perceiving benefits of physical exercise and lacking confidence in ones' exercise abilities as well as fear of injury may obstruct postoperative physical activity. This suggests that physical activity might be improved by timely targeting these cognitions.

Conflict of Interest The authors declare that they have no conflict of interest.

Open Access This article is distributed under the terms of the Creative Commons Attribution Noncommercial License which permits any noncommercial use, distribution, and reproduction in any medium, provided the original author(s) and source are credited. 


\section{References}

1. Annesi JJ. Relations of mood with body mass index changes in severely obese women enrolled in a supported physical activity treatment. Obes Facts. 2008;1:88-92.

2. Annesi JJ, Unruh JL. Relations of exercise, self-appraisal, mood changes and weight loss in obese women: testing propositions based on Baker and Brownell's (2000) model. Am J Med Sci. 2008;335:198-204.

3. Abu-Omar K, Rutten A, Lehtinen V. Mental health and physical activity in the European Union. Soz Praventivmed. 2004;49:301-9.

4. Hamer M, Stamatakis E, Steptoe A. Dose response relationship between physical activity and mental health: The Scottish Health Survey. Br J Sports Med. 2008;43:1111-4.

5. Adams TB, Moore MT, Dye J. The relationship between physical activity and mental health in a national sample of college females. Women Health. 2007;45:69-85.

6. Penedo FJ, Dahn JR. Exercise and well-being: a review of mental and physical health benefits associated with physical activity. Curr Opin Psychiatry. 2005;18:189-93.

7. Aittasalo M, Miilunpalo S, Stahl T, et al. From innovation to practice: initiation, implementation and evaluation of a physicianbased physical activity promotion programme in Finland. Health Promot Int. 2007;22:19-27.

8. Grandes G, Sanchez A, Torcal J, et al. Targeting physical activity promotion in general practice: characteristics of inactive patients and willingness to change. BMC Public Health. 2008;8:172.

9. Haverly K, Davison KK. Personal fulfillment motivates adolescentsto be physically active. Arch Pediatr Adolesc Med. 2005;159:1115-20.

10. Villanova N, Pasqui F, Burzacchini S, et al. A physical activity program to reinforce weight maintenance following a behavior program in overweight/obese subjects. Int $\mathrm{J}$ Obes (Lond). 2006;30:697-703.

11. Catenacci VA, Wyatt HR. The role of physical activity in producing and maintaining weight loss. Nat Clin Pract Endocrinol Metab. 2007;3:518-29.

12. Jones F, Harris P, Waller $\mathrm{H}$, et al. Adherence to an exercise prescription scheme: the role of expectations, self-efficacy, stage of change and psychological well-being. Br J Health Psychol. 2005; 10:359-78.

13. McGuire MT, Wing RR, Klem ML, et al. What predicts weight regain in a group of successful weight losers? J Consult Clin Psychol. 1999;67:177-85.

14. Bond DS, Phelan S, Wolfe LG, et al. Becoming physically active after bariatric surgery is associated with improved weight loss and health-related quality of life. Obesity (Silver Spring). 2009;17:78-83.

15. Bond D, Leahey TM, Vithiananthan S, et al. Bariatric surgery for severe obesity: the role of patient behavior. Med Health R I. 2009;92:58-60.

16. Leventhal H, Leventhal EA, Contrada RJ. Self-regulation, health and behavior: a perceptual-cognitive approach. Psychol Health. 1998;13:717-33.

17. Ogden J. Health psychology: a textbook. 3rd ed. Maidenhead, Berkshire, UK: Open University Press; 2004.

18. Steptoe A, Rink E, Kerry S. Psychosocial predictors of changes in physical activity in overweight sedentary adults following counseling in primary care. Prev Med. 2000;31:183-94.

19. Courneya KS, Plotnikoff RC, Hotz SB, et al. Predicting exercise stage transitions over two consecutive 6-month periods: a test of the theory of planned behaviour in a population-based sample. $\mathrm{Br}$ J Health Psychol. 2001;6:135-50.

20. Bond DS, Evans RK, DeMaria EJ, et al. Physical activity stage of readiness predicts moderate-vigorous physical activity participation among morbidly obese gastric bypass surgery candidates. Surg Obes Relat Dis. 2006;2:128-32.
21. Larsen JK, Geenen R, van Ramshorst B, et al. Binge eating and exercise behavior after surgery for severe obesity: a structural equation model. Int J Eat Disord. 2006;39:369-75.

22. Barton SB, Walker LL, Lambert G, et al. Cognitive change in obese adolescents losing weight. Obes Res. 2004;12:313-9.

23. Belachew M, Legrand MJ, Defechereux TH, et al. Laparoscopic adjustable silicone gastric banding in the treatment of morbid obesity. A preliminary report. Surg Endosc. 1994;8:1354-6.

24. Baecke JA, Burema J, Frijters JE. A short questionnaire for the measurement of habitual physical activity in epidemiological studies. Am J Clin Nutr. 1982;36:936-42.

25. Philippaerts RM, Matton L, Wijndaele K, et al. Validity of a physical activity computer questionnaire in 12- to 18-year-old boys and girls. Int J Sports Med. 2006;27:131-6.

26. Philippaerts RM, Westerterp KR, Lefevre J. Doubly labelled water validation of three physical activity questionnaires. Int J Sports Med. 1999;20:284-9.

27. Cohen J. Statistical power analysis for the behavioural sciences. 2nd ed. Hillsdale, NJ: Lawrence Erlbaum Associates; 1988.

28. Deitel M, Gawdat K, Melissas J. Reporting weight loss 2007. Obes Surg. 2007;17:565-8.

29. Larsen JK, Geenen R, van Ramshorst B, et al. Psychosocial functioning before and after laparoscopic adjustable gastric banding: a cross-sectional study. Obes Surg. 2003;13:629-36.

30. van Hout GC, Hagendoren CA, Verschure SK, et al. Psychosocial predictors of success after vertical banded gastroplasty. Obes Surg. 2009;19:701-7.

31. Wolfe BL, Terry ML. Expectations and outcomes with gastric bypass surgery. Obes Surg. 2006;16:1622-9.

32. Zijlstra H, Boeije HR, Larsen JK, et al. Patients' explanations for unsuccessful weight loss after laparoscopic adjustable gastric banding (LAGB). Patient Educ Couns. 2009;75:108-13.

33. Zijlstra H, Larsen JK, de Ridder DT, et al. Initiation and maintenance of weight loss after laparoscopic adjustable gastric banding. The role of outcome expectation and satisfaction with the psychosocial outcome. Obes Surg. 2009;19:725-31.

34. Zijlstra H, Larsen JK, van Ramshorst B, et al. The association between weight loss and self-regulation cognitions before and after laparoscopic adjustable gastric banding for obesity: a longitudinal study. Surgery. 2006;139:334-9.

35. Kinzl JF, Schrattenecker M, Traweger C, et al. Psychosocial predictors of weight loss after bariatric surgery. Obes Surg. 2006;16:1609-14.

36. Curtis P. The experiences of young people with obesity in secondary school: some implications for the healthy school agenda. Health Soc Care Community. 2008;16:410-8.

37. Ekkekakis P, Lind E. Exercise does not feel the same when you are overweight: the impact of self-selected and imposed intensity on affect and exertion. Int J Obes (Lond). 2006;30: $652-60$.

38. Leeuw M, Goossens ME, Linton SJ, et al. The fear-avoidance model of musculoskeletal pain: current state of scientific evidence. J Behav Med. 2007;30:77-94.

39. Vlaeyen JW, Linton SJ. Fear-avoidance and its consequences in chronic musculoskeletal pain: a state of the art. Pain. 2000;85: 317-32.

40. Wouters EJ, van Nunen AM, Vingerhoets AJ, et al. Setting overweight adults in motion: the role of health beliefs. Obes Facts. 2009;2:362-9.

41. Elfving B, Andersson T, Grooten WJ. Low levels of physical activity in back pain patients are associated with high levels of fear-avoidance beliefs and pain catastrophizing. Physiother Res Int. 2007;12:14-24.

42. Wouters EJ, Van Nunen AM, Geenen R, et al. Effects of aquajogging in obese adults: a pilot study. J Obes. 2010; Article ID 231074 